\title{
Erratum to: Steroid-resistant nephrotic syndrome in a child with dysmorphic features: Questions
}

\author{
Osama Y. Safdar ${ }^{1} \cdot$ Sherif M. El-Desoky ${ }^{1}$ • Detlef Bockenhauer ${ }^{2} \cdot$ Neil Sebire $^{3}$ • \\ Jameela A. Kari ${ }^{1}$
}

Published online: 20 April 2016

(C) IPNA 2016

\section{Erratum to: Pediatr Nephrol}

DOI: 10.1007/s00467-014-2752-4

The name of the first author was rendered wrongly; his correct name is Osama Y. Safdar. The same error occurred in a previous erratum to this article (DOI: 10.1007/s00467-014-2783-x), which corrected the name of the fourth author, Neil Sebire, and the wording of the legend of Fig. 2 ("Renal biopsy with glomeruli stained with silver stain").

1 Pediatric Nephrology Unit, Department of Pediatrics, Faculty of Medicine, King Abdulaziz University, PO Box 80215,

Jeddah 21589, Kingdom of Saudi Arabia

2 Renal Unit, University College London, London, UK

3 Department of Pathology, Great Ormond Street Hospital, Institute of Child Health, University College London, London, UK 